\title{
MÉTODO DE CASOS PARA PROMOVER EL PENSAMIENTO CRÍTICO DE ESTUDIANTES DE DERECHO EN ENTORNOS VIRTUALES DE APRENDIZAJE
}

\author{
Quezada Castro María del Pilar ${ }^{1}$, Castro Arellano María del Pilar², Christian Abraham Dios Castillo ${ }^{3}$, Gallo \\ Aguila Carlos Ignacio ${ }^{4}$, Oliva Núñez Juan Manuel $^{5}$ y Quezada Castro Guillermo Alexander ${ }^{6}$ \\ C20853@utp.edu.pe,m_castro_a@uap.edu.pe, cdios@utp.edu.pe, c_gallo_a@doc.uap.edu.pe,j_oliva@doc.uap. \\ edu.pe,g_quezada_ca@doc.uap.edu.pe \\ ORCID $^{1}$ : https://orcid.org/0000-0002-1012-570X, ORCID²: https://orcid.org/0000-0002-6661-9928, \\ ORCID $^{3}$ : https://orcid.org/0000-0002-2469-9237, ORCID ${ }^{4}$ : https://orcid.org/0000-0003-1382-0545, \\ ORCID ${ }^{5}$ : https://orcid.org/0000-0001-9319-3689, ORCID ${ }^{6}$ : https://orcid.org/0000-0002-4868-1664 \\ Universidad Tecnológica del Perú, Piura ${ }^{1,3}$ \\ Universidad César Vallejo, Piura ${ }^{4}$ \\ Universidad Alas Peruanas, Piura ${ }^{2}, 6$ \\ Piura-Perú
}

Recibido (04/06/20), Aceptado (18/06/20)

\begin{abstract}
Resumen: La actividad universitaria desarrollada en los entornos virtuales de aprendizaje requiere la aplicación de estrategias destinadas a potencializar la relación docente - estudiante, para lo cual se planteó como objetivo determinar la aceptación del método de casos para promover el pensamiento crítico, desde la perspectiva del docente universitario. La metodología se sustentó en el paradigma positivista, enfoque cuantitativo, diseño no experimental, tipo descriptivo y método analítico. Se aplicó un cuestionario a 43 docentes universitarios encargados de una asignatura adscrita a la carrera profesional de Derecho y con vínculo laboral con universidades de la Región Piura-Perú. Se obtuvo como resultado que el $84 \%$ de los participantes consideraron que el pensamiento crítico se promueve a partir de la aplicación del método de casos y que el aprendizaje es más efectivo si los estudiantes construyen o descubren el conocimiento a partir interacción docente-estudiante. Se concluyó que el método de casos es reconocido y aceptado por los docentes en las facultades de Derecho porque incentiva el análisis y reflexión del estudiante, quienes al analizar los hechos deberán plantear una alternativa de solución.
\end{abstract}

Palabras Clave: Pensamiento crítico, docente virtual, método de casos, competencias.

\section{CASE METHOD FOR PROMOTING CRITICAL THINKING OF LAW STUDENTS IN VIRTUAL LEARNING ENVIRONMENTS}

\begin{abstract}
The university activity developed in virtual learning environments requires the application of strategies aimed at potentiating the teacher-student relationship, for which purpose it was proposed to determine the acceptance of the case method to promote critical thinking, from the perspective of the university teacher. The methodology was based on the positivist paradigm, quantitative approach, nonexperimental design, descriptive type and analytical method. A questionnaire was applied to 43 university professors in charge of a subject assigned to the professional career of Law and with an employment relationship with universities in the Piura-Peru Region. As a result, $84 \%$ of the participants considered that critical thinking is promoted through the application of the case method and that learning is more effective if students build or discover knowledge from teacher-student interaction. It was concluded that the case method is recognized and accepted by teachers in law schools because it encourages the analysis and reflection of the student, who when analyzing the facts must propose an alternative solution.
\end{abstract}

Keywords: Critical thinking, virtual teacher, case method, competencies. 


\section{I.INTRODUCCIÓN}

La percepción tradicional del docente universitario se centró en un salón de clases, en el cual la interacción de estudiantes se consolidó como el resultado de la aplicación de estrategias vinculadas a una participación activa materializada en una calificación contenida en el registro académico presentado en la respectiva facultad.

Las universidades peruanas, a consecuencia de la pandemia identificada como "COVID-19" (coronavirus), adoptaron la decisión de incursionar públicamente en entornos virtuales de aprendizaje, ello con la finalidad de continuar brindando el servicio educativo a favor de estudiantes.

La interacción en entornos virtuales de aprendizaje originó que tanto docentes como estudiantes, a pesar de las circunstancias, adecuaran su tiempo y recursos logísticos para aprender de modo sincrónico o asincrónico en sesiones virtuales correspondientes a su carrera profesional, de tal forma, que el contexto requería la evolución del docente tradicional a un docente virtual.

En tal sentido, la pedagogía en la carrera profesional de Derecho, debía experimentar el uso de métodos aplicables a las plataformas virtuales que permitan al estudiante reconocer el dinamismo e interacción en el mundo digital, para lo cual era necesario determinar la aceptación del método de casos para promover el pensamiento crítico, desde la perspectiva del docente universitario, siendo ello el contexto de la presente investigación.

\section{II.DESARROLLO}

El método de casos permite la integración progresiva con un enfoque multidisciplinario a través del desarrollo de competencias profesionales [1], razón por la cual es adecuado para la enseñanza universitaria [2], toda vez que promueve analizar los hechos y propone una alternativa de solución vinculada al pensamiento crítico [3], materializándose como una herrmienta para evaluar la información [4].

La información derivada de cada asignatura es recepcionada por los estudiantes, actividad que es complementaria al desarrollo de habilidades y competencias [5], siendo que en el caso universitario, se evidencia una estructura lógica y de principios éticos [6], que sustentan una evaluación consciente y crítica [7].

El pensamiento crítico pretende formar en el estudiante características como la flexibilidad, prudencia y tolerancia [8], identificación de dificultades [9], comprensión de problemas [10], actuación coherente [11], resolución de problemas [12], las cuales fortalecerán el perfil profesional [13], enriquecido por la educacion científica [14].
La educación científica deberá establecer líneas de acción atractivas que viabilicen la interacción de los estudiantes en entornos virtuales [15], siendo en el caso de la carrera profesional de Derecho que deberá implementar estrategias que incidan en una comunicación directa con predominio del razonamiento analítico que difunda la fundamentación crítica en la formación de futuros egresados con proyección social, a través del uso de modelos de transmisión [16], esencia que deberá ser asumida por la enseñanza virtual.

La enseñanza virtual se encuentra vinculada con la implementación de la tecnología de información y comunicación [17]; sin embargo, se debe tener en cuenta que la sola presencia de la tecnología no asegura el aprendizaje [18], razón por la cual es necesario implementar actividades pedagógicas que asocien el uso de una metodología eficaz en el proceso enseñanza-aprendizaje [19], verificable para su mejor desempeño [20], en la arquitectura tecnológica proporcionada por los entornos virtuales de aprendizaje [21].

Los entornos virtuales de aprendizaje representan un espacio de afinidad entre docentes y estudiantes [22], tienen por finalidad crear un espacio afectivo sustentado en el apoyo mutuo [23], es decir, no debe expresar competencia entre los participantes, al contrario, representa un desafío en la enseñanza universitaria [24], que deberá asumir los retos sociopolíticos y económicos que se presentarán [25], aunque para ello es necesario potencializar el autoestudio [26].

El autoestudio simboliza la iniciativa del estudiante por interactuar en los entornos virtuales de aprendizaje, para ello las instituciones de educación superior desarrollarán competencias digitales que permitan comprender eficientemente las tecnologías y el acceso a la información disponible en internet [27], lo cual es factible, a través de la creatividad [28] y bajo la masificación de las redes sociales académicas [29] en beneficio de la población.

\section{III.METODOLOGÍA}

La presente investigación se sustentó en el paradigma positivista, enfoque cuantitativo, diseño no experimental, tipo descriptivo y método analítico. La muestra seleccionada por conveniencia estuvo representada por 43 docentes (abogados, ingenieros y administradores) encargados de asignaturas, contenidas en un entorno virtual de aprendizaje, pertenecientes a la carrera profesional de Derecho y que tienen vínculo laboral vigente con universidades ubicadas en la Región Piura - Perú. Se desarrolló un cuestionario de 14 ítems, el cual obtuvo el coeficiente de Alfa de Cronbach de 0.824, siendo la información obtenida procesada mediante el software 
estadístico SPPS versión 24 y presentada mediante microsoft excel.

\section{IV.RESULTADOS}

En la presente investigación los docentes aceptaron participar voluntariamente, toda vez que desde su perspectiva reconocen la existencia del método de casos que podría ser aplicado a los entornos virtuales de aprendizaje relacionados con las asignaturas de la carrera profesional de Derecho, para ello, el equipo investigador consideró agrupar los resultados, conforme se aprecia a continuación:

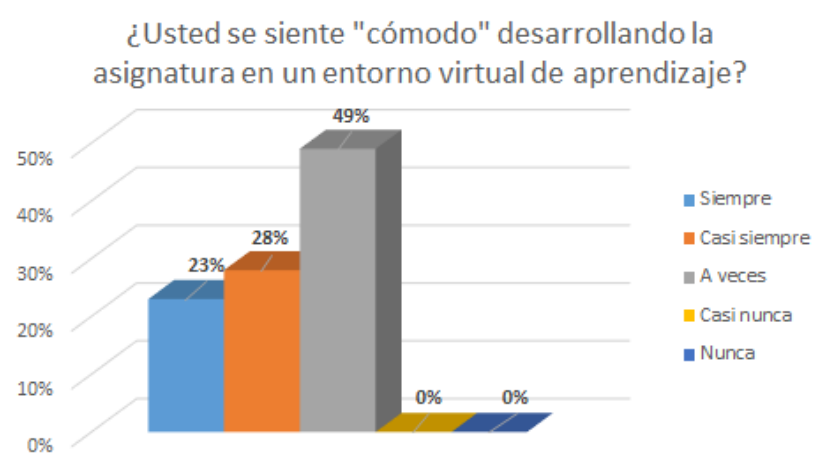

Figura 1. Percepción del entorno virtual de aprendizaje

De la figura 1, es necesario deducir que la virtualidad y la docencia en universidades peruanas está en proceso de consolidación, a consecuencia de la pandemia (coronavirus) la relación docente - estudiante se trasladó desde la concepción tradicional de un salón de clases a una plataforma virtual y con ello se derivarán futuras investigaciones enmarcadas en describir o analizar el desenvolvimiento de los protagonistas.

\section{¿Considera que el estudiante se adaptará a un entorno virtual de aprendizaje?}

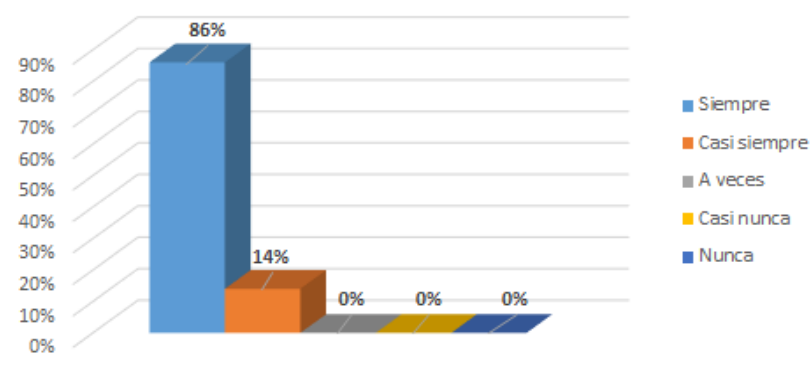

Figura 2. Percepción de la fidelización del entorno virtual de aprendizaje

De la figura 2, se deduce que si bien es cierto los universitarios están identificados con el uso de la tec- nología; sin embargo, se advierte que podrían existir estudiantes, a quienes por sus características sociales, económicas o culturales se encuentren en pleno proceso de adaptación, situación que podría ser analizada con detenimiento en una futura investigación.

\section{¿Considera que el entorno virtual de aprendizaje permite la interacción entre estudiante - docente?}

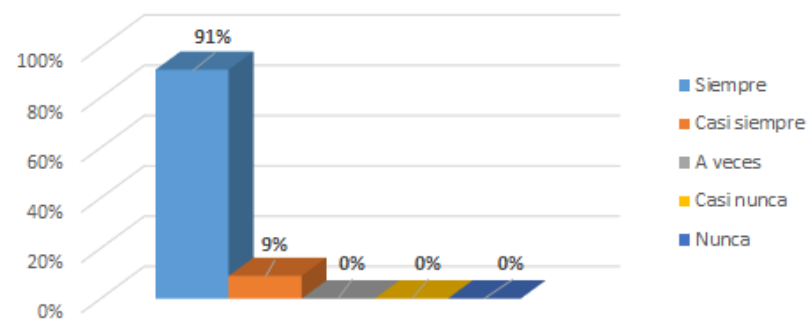

Figura 3. Percepción de la interacción entre estudiante - docente

De la figura 3, se debe tener en cuenta que los entornos virtuales de aprendizaje requieren ciertos conocimientos que viabilicen la interacción, situación que está relacionada a las estrategias universitarias para desarrollar una convivencia de paz, lo cual sería factible a través de códigos de ética que regulen la intervención en el ciberespacio.

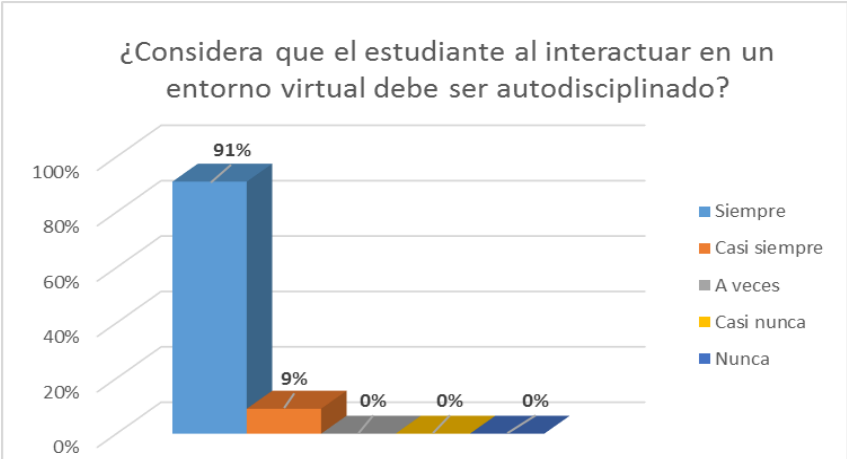

Figura 4. Percepción de la participación del autoestudio

De la figura 4, se debe tener en cuenta que desde la percepción del docente, la autodisciplina podría ser una habilidad y característica de las personas que incursionan en un mundo virtual, por lo que sería necesario conocer anticipadamente las ventajas y desventajas, situación que podría ser factible ser analizada en futuras investigaciones. 
¿Considera que el método de casos sería aplicable en estudiantes de Derecho que interactúan en entornos virtuales de aprendizaje?

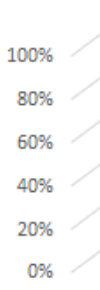

Figura 5. Percepción de la aplicación de la aplicación del método de casos

De la figura 5, se debe tener en cuenta que el método de casos promueve capacidades del estudiante que estarían vinculadas con el pensamiento crítico, es decir, se deduce que la aplicación del referido método sería ideal implementarlo en el uso de plataformas virtuales, porque se enfocaría en desarrollar la creatividad e innovación del estudiante universitario.

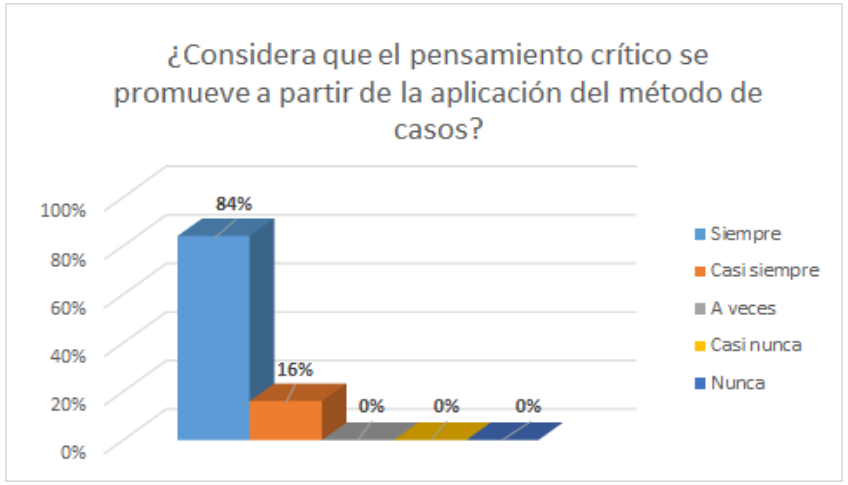

Figura 6. Percepción de la aplicación de pensamiento crítico

De la figura 6, se deduce que existiría una conexión de interdependencia entre pensamiento crítico y método de casos, lo cual podría ser beneficioso para el proceso de enseñanza-aprendizaje, el mismo que al desarrollarse en un entorno virtual permitiría al estudiante de Derecho desarrollar capacidades que enriquecerían su formación como futuro profesional.
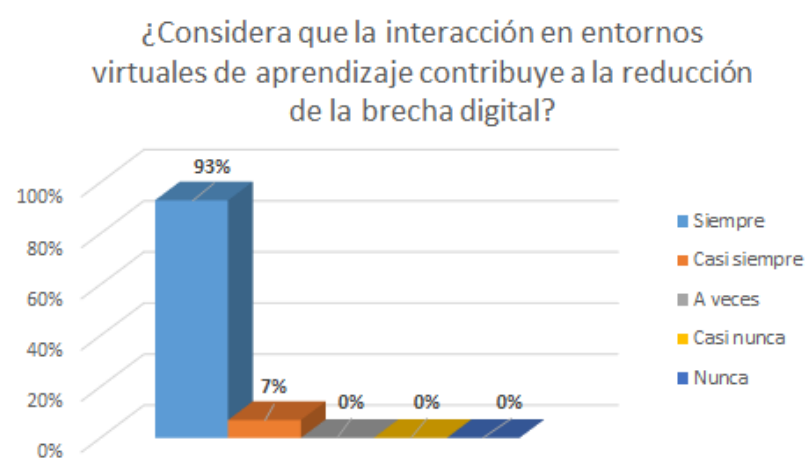

\section{Figura 7. Percepción de la reducción de la brecha digital}

De la figura 7, se debe tener en cuenta que la existencia de los entornos virtuales de aprendizaje promueven el dinamismo e incentivan a incursionar en las tecnologías de información y comunicación, situación que originaría mayor contacto con la realidad digital, siendo necesario analizar las consecuencias de lo descrito en futuras investigaciones.

En ese orden de ideas, se aprecia que el análisis de casos permite a los participantes la visualización de realidades en contextos similares o diferentes al entorno cercano en el que se desarrollan; de esta manera, se identificará similitudes y particularidades de los casos estudiados con los experimentados o posibles de experimentar; asimismo, el uso de entornos virtuales permite una interacción dinámica entre los participantes del proceso de estudio de casos, al reducir o eliminar la brecha presencial y de disponibilidad de tiempo para el pronunciamiento de comentarios o debates. Sin embargo, adquiere una especial relevancia el análisis de los comentarios y participaciones de los estudiantes, en la medida que el moderador exija su fundamentación a través de citas bibliográficas y comentarios analíticos, lo cual es precisamente el resultado de la interacción de ideas fundamentadas y el análisis de su aplicabilidad a los casos de estudio, lo que fomenta el aprendizaje colaborativo.

Finalmente, es necesario determinar estrategias gubernamentales que garanticen la continuidad de los estudios superiores en una cuarentena, para lo cual el docente implementará estrategias pedagógicas adecuadas a entornos virtuales, en beneficio del servicio educativo, lo cual es factible ser profundizado en futuras investigaciones. 


\section{V.CONCLUSIONES}

1.El método de casos es reconocido y aceptado por los docentes en las facultades de Derecho de las universidades peruanas, toda vez que incentiva el análisis, motivación y reflexión en el estudiante sobre casos derivados de la realidad, lo cual contribuirá a su proceso de formación como futuro profesional.

2.El método de casos podría ser aplicado de manera prioritaria en la interacción docente - estudiante realizada en entornos virtuales de aprendizaje porque se relacionaría con el desarrollo de las habilidades contempladas en el proceso enseñanza - aprendizaje.

3.El docente virtual se identifica con el uso del método de casos en estudiantes de Derecho porque permite promover el pensamiento crítico, exponer los hechos y plantear una alternativa de solución para una situación compleja o para un caso específico.

4.El docente participante del entorno virtual de aprendizaje considera que el estudiante debe ser autodisciplinado, máxime si se aprecia la predisposición de acceder a las tecnologías de información y comunicación, por lo tanto, correspondería analizar el perfil social y académico en futuras investigaciones.

5.El pensamiento crítico implementado en el entorno virtual de aprendizaje implica el análisis de una situación en su conjunto que contribuiría a la fidelización del estudiante de Derecho, en consecuencia, es necesario conocer dentro de qué contexto se realizará y qué estrategias estarían destinadas a la captación del público con respecto a la carrera profesional referida.

\section{REFERENCIAS}

[1]C. De la Fe Rodríguez, I. Vidaurreta Porrero, Á. Gómez Martín y J. C. Corrales Romero, «El método de estudio de casos: Una herramienta docente válida para la adquisición de competencias,» Revista Electrónica Interuniversitaria de Formación del Profesorado, vol. 18, no 3, pp. 127-137, 2015.

[2]A. Estrada Cuzcano y K. L. Alfaro Mendives, «E1 método de casos como alternativa pedagógica para la enseñanza de la bibliotecología y las ciencias de la información,» Revista Investigación Bibliotecológica, vol. 29, nº 65, pp. 195-211, 2015.

[3]M. A. Montiel Galindo, D. G. Charles Estrada y S. L. Olivares Olivares, «Método de casos como estrategia didáctica para desarrollar el pensamiento crítico en estudiantes de turismo,» Revista Ciencia, Docencia y Tecnología , vol. 29, nº 57, pp. 88-110, 2018.

[4]M. Vernier, L. Cárcamo y E. Scheihing, «Pensamiento crítico de los jóvenes ciudadanos frente a las noticias en Chile,» Revista Comunicar, vol. XXVI, $\mathrm{n}^{\circ}$ 54, pp. 101-110, 2018.
[5]E. J. Steffens, D. d. C. Ojeda, J. . L. Martínez, H. G. Hernández y Y. H. Moronta, «Presencia del pensamiento crítico en estudiantes de educación superior de la Costa Caribe Colombiana,» Revista Espacios, vol. 39, $\mathrm{n}^{\circ} 30$, pp. 1-14, 2018.

[6]Y. E. Loaiza Zuluaga y L. D. Osorio, «El desarrollo de pensamiento crítico en ciencias naturales con estudiantes de básica secundaria en una Institución Educativa de Pereira - Risaralda,» Revista Diálogos sobre Educación, vol. 9, n 16, pp. 1-24, 2018.

[7]P. Morales Bueno, «Aprendizaje basado en problemas (ABP) y habilidades de pensamiento crítico ¿una relación vinculante?,» Revista Electrónica Interuniversitaria de Formación del Profesorado, vol. 21, n 2, pp. 91-108, 2018.

[8]J. L. Valencia Castro, S. Tapia Vallejo y S. L. Olivares Olivares, «La simulación clínica como estrategia para el desarrollo del pensamiento crítico en estudiantes de medicina,» Revista Investigación en Educación Médica, vol. 8, n 29, pp. 13-22, 2019.

[9]M. E. Godoy Zúñiga y K. M. Calero Cedeño, «Pensamiento crítico y tecnología en la educación universitaria. Una aproximación teórica,» Revista Espacios, vol. 39, n 25, pp. 1-6, 2018.

[10]E. García Carpintero Blas, J. Siles González, E. Martínez Roche, E. Martínez de Miguel y C. Manso Perea, «Percepciones de los estudiantes sobre el desarrollo del pensamiento crítico-reflexivo a través del portafolio de prácticas,» Revista Investigación en Enfermería: Imagen y Desarrollo, vol. 20, no 1, pp. 1-12, 2018.

[11]F. H. Prieto Galindo, «El pensamiento crítico y autoconocimiento,» Revista de Filosofía, vol. 74, pp. 173191, 2018.

[12]E. Leonor Zambrano, «Prácticas pedagógicas para el desarrollo de competencias ciudadanas,» Revista Electrónica de Investigación Educativa, vol. 20, $\mathrm{n}^{\mathrm{o}}$ 1, pp. 69-82, 2018.

[13]E. Zelaieta Anta y I. C. Ortiz de Barrón, «El desarrollo del pensamiento crítico en la formación inicial del profesorado: Análisis de una estrategia pedagógica desde la visión del alumnado,» Revista de currículum y formación del profesorado, vol. 22, nº 1, pp. 197-214, 2018.

[14]V. Ortega-Quevedo y C. Gil Puente, «La naturaleza de la ciencia y la tecnología. Una experiencia para desarrollar el pensamiento crítico,» Revista Científica, vol. 35, no 2, pp. 167-182, 2019.

[15]J. J. Sánchez Miranda, A. González Polo y A. Monroy Rodríguez, «La formación de docentes normalistas: De la tradición pedagógica a los entornos virtuales de aprendizaje,» Revista Iberoamericana para la Investigación y el Desarrollo Educativo, vol. 10, n 19, pp. 1-30, 
2019.

[16]S. Osuna Acedo, «Didáctica y espejismos de las plataformas virtuales,» Revista Educação e Cultura Contemporânea, vol. 6, nº 13, pp. 23-34, 2009.

[17]C. Cavadía, F. Payares, K. Herrera, J. Jaramillo y L. Meza, «Los entornos virtuales de aprendizaje como estrategia de mediación pedagógica,» Revista Aglala, vol. 10, no 2, pp. 212-220, 2019.

[18]A. C. Urquidi Martin, M. S. Calabor Prieto y C. Tamarit Aznar, «Entornos virtuales de aprendizaje: modelo ampliado de aceptación de la tecnología,» Revista Electrónica de Investigación Educativa, vol. 21, pp. 1-12, 2019.

[19]R. J. Ayala Pezzutti, C. M. Laurente Cárdenas, C. D. Escuza Mesías, L. A. Núñez Lira y J. R. Díaz Dumont, «Mundos virtuales y el aprendizaje inmersivo en educación superior,» Revista Propósitos y Representaciones, vol. 8, $\mathrm{n}^{\circ} 1$, pp. 1-17, 2020.

[20]E. Rodríguez Hernández y G. Vega Cruz, «Estudio de la necesidad del uso de las TIC para la capacitación enel tema Gestión de Inventarios desde el puesto de trabajo,» Revista Pedagógica, vol. 7, n 2, pp. 168-190, 2019.

[21]G. Tapia-Repetto, C. Gutierrez y O. Tremillo-Maldonado, «Nuevas tecnologías en educación superior. Estudio de percepción en estudiantes acerca del uso de WhatsApp y Entornos Virtuales de Aprendizaje (Plataforma Moodle),» Revista Odontoestomatología, vol. XX, nº 33, pp. 37-43, 2019.

[22]R. Ponce Carrillo y L. M. Alarcón Pérez, «Entornos virtuales para la escritura académica. Un modelo en Minecraft,» Revista Alteridad, vol. 15, $\mathrm{n}^{\mathrm{o}} 1$, pp. 76-87, 2020 .

\section{RESUMEN CURRICULAR}

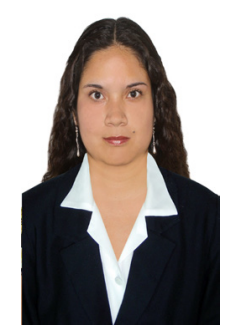

María del Pilar Quezada Castro Abogada, Maestro en Derecho Civil. Bachiller en Turismo, Hotelería y Gastronomía. Árbitro. Conciliadora Extrajudicial. Doctorando en Derecho en la Universidad Nacional de Piura. Doctorando en Educación en la Universidad César Vallejo. Maestrista en Derecho de la Empresa en la Escuela de Postgrado Neumann. Docente en la Universidad Tecnológica del Perú, Universidad Alas Peruanas y Universidad Privada Antenor Orrego, sede Piura, correo electrónico: $\underline{\text { C20853@utp.edu.pe. }}$
[23]E. d. J. García Guardado, «La interactividad en espacios de aprendizaje virtuales: roles de profesores y estudiantes,» Daena: International Journal of Good Conscience, vol. 14, no 1, pp. 5-29, 2019.

[24]Y. Ocaña-Fernández, L. Valenzuela-Fernández y J. Morillo-Flores, «La competencia digital en el docente universitario,» Revista Propósitos y Representaciones, vol. 8, $\mathrm{n}^{\mathrm{o}}$ 1, pp. 1-13, 2020.

[25]G. A. Santana Sardi y J. A. Vigueras Moreno, «Hacia un Sistema Virtual de orientación vocacional,» Revista Cubana de Educación Superior, vol. 38, no 3, pp. $1-15,2019$.

[26]A. F. Contreras-Colmenares y L. M. Garcés-Díaz, «Ambientes Virtualesde Aprendizaje: dificultades de uso en los estudiantes de cuarto grado de primaria,» Prospectiva.Revista de Trabajo Social e intervención social, no 27, pp. 215-240, 2019.

[27]L. T. Gabriela Vilanova y J. Varas, «Desarrollo de competencias digitales en propuestas pedagógicas en ambientes mediados. Un caso en educación superior bajo modelo de aula extendida,» Revita Informes Cientificos Técnicos - UNPA, vol. 11, nº 3, pp. 61-87, 2019. [28]E. E. Espinoza Freire, N. V. Ley Leyva y V. J. Guamán Gómez, «Aprendizaje cooperativo y la Web 2.0. Universidad,» Revista Ibérica de Sistemas e Tecnologias de Informação, $\mathrm{n}^{0}$ 25, pp. 520-538, 2020.

[29]D. Calderón-Garrido, A. León-Gómez y R. Gil-Fernández, «El uso de las redes sociales entre los estudiantes de grado de maestro en un entorno exclusivamente online,» Vivat Academia. RevistadeComunicación, $\mathrm{n}^{\mathrm{o}}$ 147, pp. 23-40, 2019.

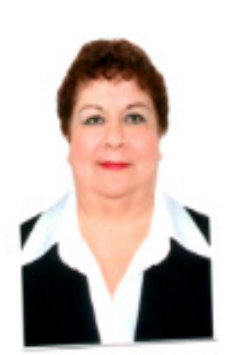

María del Pilar Castro Arellano Abogada. Licenciada en Trabajo Social. Maestro en Derecho Civil. Doctor en Derecho. Egresada de la Maestría de Administración y Dirección de Empresas. Bachiller en Turismo, Hotelería y Gastronomía. Docente universitario. Coordinadora Académica de la Escuela Profesional de Turismo, Hotelería y Gastronomía de la Universidad Alas Peruanas, filial Piura - Perú, correo electrónico: m_castro_a@uap.edu.pe 
Christian Abraham Dios Castillo Ingeniero de Sistemas. Asesor en Auditoría de Administración de Sistemas y Tecnologías de Información. Master in Business Administration MBA. Asesor en Planeación y Organización, implementación de Tableros de Comando en empresas privadas y organizaciones gubernamentales. Doctor en Administración de la Educación. Asesor de Universidades en la implantación del sistema de administración de investigaciones. Consultor especialista en gestión de proyectos de tecnologías de información en organizaciones diversas, y estrategias de e-learning. Director de Investigación. Universidad Tecnológica Del Perú. Región Norte, correo electrónico: cdios@utp.edu.pe

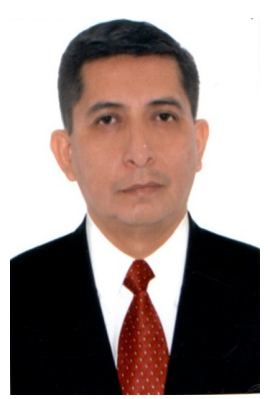

\section{Carlos Ignacio Gallo Aguila Ingeniero} Industrial, Maestro en Docencia Universitaria y Gestión Educativa, Doctor en Ciencias de la Educación. Docente en la Universidad César Vallejo, Piura, correo electrónico: cgalloa@gmail.com

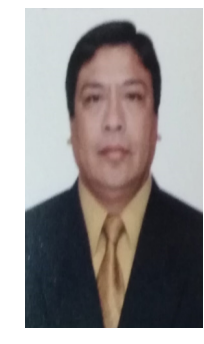

Juan Manuel Oliva Núñez Arquéologo, Maestro en Educación con mención en Investigación y Docencia, Doctor en Ciencias de la Educación. Coordinador de la oficina de Investigación, Innovación y Emprendimiento de la Universidad Alas Peruanas, Piura. Docente a tiempo completo en la Universidad Alas Peruanas, Perú - filial Piura, correo electrónico: __oliva@doc.uap.edu.pe.

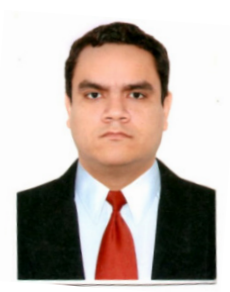

Guillermo Alexander Quezada Castro Abogado, Maestro en Derecho Civil, egresado del Doctorado en Derecho y Maestría en Derecho Constitucional por la Universidad Alas Peruanas. Egresado de la Maestría en Gestión Pública por la Universidad San Pedro. Egresado de la Maestría en Ingeniería con mención en Dirección y Gestión de Proyectos en la Universidad Católica de Trujillo. Maestrista en Derecho a la Empresa por la Escuela de Posgrado Neumann. Docente a tiempo completo en la Universidad Alas Peruanas, Perú -filial Piura, correo electrónico: g_quezada_ca@doc.uap.edu.pe. 